\title{
Taxonomic Confusion of Pseudomonas cissicola Originated from Mislabeling by International Type Culture Collections
}

\author{
M. GOTO \\ Faculty of Agriculture, Shizuoka University, 836 Ohya, Shizuoka 422, Japan
}

\begin{abstract}
The type strain (strain ICMP 4289) and two reference strains (strains ICMP 4290 and ICMP 4291) of Pseudomonas cissicola (Takimoto 1939) Burkholder 1948 were obviously mislabeled at the Plant Disease Division Culture Collection (presently the International Collection of Microorganisms from Plants) in New Zealand when they were deposited in 1975 or subsequently when they were coded for the catalog of the collection. The strains were also mislabeled at the National Collection of Plant Pathogenic Bacteria in England when strain NCPPB $2982^{T}$ ( $T=$ type strain) was transferred from the Plant Disease Division Culture Collection. Recently, these errors have caused considerable confusion in the taxonomic placement of this bacterium, not only in workers who received cultures from the culture collections but also in workers who are directly responsible for the culture collections. The type strain of the bacterium should be strain PC1 in the culture collection of plant-pathogenic bacteria at the Laboratory of Plant Pathology, Shizuoka University, strain ATCC 33616 in the American Type Culture Collection, or strain CCM 2888 in the Czechoslovak Collection of Microorganisms.
\end{abstract}

In this paper I describe the background of the recent taxonomic confusion concerning Pseudomonas cissicola (Takimoto 1939) Burkholder 1948, the causal agent of bacterial leaf spot of an ivy weed, Cayratia japonica (Thunb.) Gagn., and advise the curators of international type culture collections that fundamental errors should be eliminated to protect the reputations of their cultures in terms of identification and purity.

Recent publications related to the taxonomy of $P$. cissicola. In two papers recently published in the International Journal of Systematic Bacteriology, workers have referred to the taxonomic relationship between $P$. cissicola and other nonfluorescent plant-pathogenic pseudomonads. In 1985, De Vos et al. (1) reported, on the basis of levels of rRNA similarity, that $P$. cissicola $\mathrm{NCPPB} 2982^{\mathrm{T}}$ ( $\mathrm{T}=$ type strain) and PDDCC 4290) are not related to any of four rRNA branches of the genus Pseudomonas. In 1991, Hu et al. (4) described the taxonomic affiliation of this bacterium and stated that "an examination of the flagellar insertion of strains ICMP $4289^{\mathrm{T}}$, ICMP 4290 and ICMP 4291 showed that these organisms are all motile by means of peritrichous flagella, which is consistent with identification as Agrobacterium sp. These strains should be allocated to Agrobacterium sp., until identification to species can be made. The type strain of the taxon is strain ICMP 4289 (= NCPPB 2982) and not strain ICMP 8561, which was incorrectly identified as the type."

These articles, particularly the latter, are responsible for critical confusion concerning the identification of the type strain, as well as the taxonomic status, of $P$. cissicola.

Background of the confusion. $P$. cissicola was originally isolated by Takimoto in Japan and was identified as Aplanobacter cissicola in 1939 (11). This species was transferred to the genus Pseudomonas by Burkholder in 1948 (12). Unfortunately, however, the original strains had not been maintained in any culture collection. Therefore, $P$. cissicola was included in 1974 in Addendum I of Dye's paper (2), which listed plant-pathogenic pseudomonads that were nomenspecies and had no taxonomic standing because their descriptions were inadequate and/or no cultures were available.

In the summer of 1974, after Dye's paper was submitted to the Review of Plant Pathology, coworkers and I found bacterial leaf spot of $C$. japonica in Japan and isolated the pathogenic bacterium. After pathogenicity was confirmed and bacteriological characteristics were determined, three lyophilized cultures, including the neotype strain (strain PC1), were deposited in the Culture Collection of the Plant Disease Division (PDDCC), Department of Scientific and Industrial Research, Auckland, New Zealand, in July 1975 at the request of D. W. Dye. Consequently, $P$. cissicola was added to the Approved Lists of Bacterial Names (9).

In the preprint edition of the PDDCC catalog issued in 1978 (6), the three cultures mentioned above were listed as strains PDDCC 4289 , PDDCC 4290, and PDDCC 4291 (strains $\mathrm{PC1}^{\mathrm{T}}, \mathrm{PC}$, and $\mathrm{PC} 3$, respectively).

After the description of this bacterium was published in 1977 (3), cultures of strains $\mathrm{PCl}^{\mathrm{T}}$ and PC2 were deposited in August 1977 in the Czechoslovak Collection of Microorganisms (CCM; curator, M. Kocur, Masaryk University, Brno, Czechoslovakia) and were designated strains CCM $2888^{\mathrm{T}}$ and CCM 2889, respectively.

In October 1983, I received a letter from the curator of the American Type Culture Collection (ATCC), notifying me that type strain NCPPB 2982 of $P$. cissicola, which had been transferred from National Collection of Plant Pathogenic Bacteria (NCPPB), Harpenden, England, in 1982, was peritrichous and requesting a subculture for comparison (8). No culture of this bacterium had ever been deposited in the NCPPB by me, however, strain NCPPB $2982^{\mathrm{T}}$ was reported to be the same as strain ICMP $4289^{\mathrm{T}}$ by Hu et al. (4). This was the first indication that there are problems in the identification of cultures of $\boldsymbol{P}$. cissicola that are in international culture collections.

I immediately asked the curator of the NCPPB to examine the flagellation of strain NCPPB $2982^{\mathrm{T}}$. He replied that this strain had a single polar flagellum but differed from the original description of the species in hydrolysis of starch and egg yolk (10). He sent me one ampoule of strain NCPPB $2982^{\mathrm{T}}$ for reference. This culture was immediately checked and was found to differ from strain $\mathrm{PCl}^{\mathrm{T}}$ as he had stated.

Copies of the letter of inquiry to the NCPPB described 
above were also sent at the same time to the curators of the PDDCC and the ATCC. In response the curators of both type culture collections wrote to me and asked me to send replacements for the neotype strain. Lyophilized cultures of neotype strain $\mathrm{PCl}$ obtained from the original lyophilization batch in the culture collection at the Laboratory of Plant Pathology at Shizuoka University were sent to both the PDDCC and the ATCC in early 1984.

The curators of the PDDCC and the ATCC have subsequently informed me that these cultures were deposited in the lyophilized state as strains PDDCC $8561^{\mathrm{T}}$ and ATCC $33616^{\mathrm{T}}$, respectively. At the same time the curator of the ATCC sent me two ampoules for a depositor approval check. One of these tubes was immediately checked and was confirmed to be identical to type strain $\mathrm{PC1}$, and this confirmation was reported to the ATCC.

The catalog of the International Collection of Microorganisms from Plants (ICMP), Plant Disease Division, New Zealand Department of Scientific and Industrial Research, Auckland, New Zealand (7), distributed in 1988, surprisingly listed three strains of this bacterium, including the replaced culture of strain ICMP (= PDDCC) 8561 with the supplementary remark "pleiomorphic organism."

After the publication of the paper by $\mathrm{Hu}$ et al. (4), I carefully checked the identification of the organisms in another of the ampoules of strain ATCC $33616^{\mathrm{T}}$ used for the depositor approval check in 1984 and two lyophilized cultures of strain $\mathrm{PC1}^{\mathrm{T}}$ which were taken from the same batches as those originally deposited in the PDDCC in July 1975 and in the three culture collections mentioned above in February 1984. The three cultures were in accordance with the original descriptions (3) in flagellation and the capacity to hydrolyze starch and egg yolk. Subsequently, two cultures that were deposited in 1977 in the CCM (strains CCM $2888^{\mathrm{T}}$ and CCM 2889) were checked for identification and purity and were found to be identical to the original cultures.

Critique of the reliability of identification of bacterial strains in type culture collections. The complicated situation described above can be diagrammed as follows:

$\mathrm{PC1}^{\mathrm{T}} \rightarrow \mathrm{PDDCC}(=\mathrm{ICMP}) 4289^{\mathrm{T}}$ (deposited in 1975 but not approved; reported to be peritrichous [4])

$\mathrm{PCI}^{\mathrm{T}} \rightarrow \mathrm{CCM} 2888^{\mathrm{T}}$ (deposited in 1977 and approved in 1992)

$\mathrm{PCl}^{\mathrm{T}} \rightarrow$ PDDCC (= ICMP) $4289^{\mathrm{T}} \rightarrow$ NCPPB $2982^{\mathrm{T}}$ (polarly flagellated strain with different bacteriological traits [10]) $\mathrm{PC1}^{\mathrm{T}} \rightarrow$ PDDCC (= ICMP) $4289^{\mathrm{T}} \rightarrow$ NCPPB $2982^{\mathrm{T}} \rightarrow$ ATCC $33616^{\mathrm{T}}$ (shown to be peritrichous (8); discarded in 1984)

$\mathrm{PC1}^{\mathrm{T}} \rightarrow \mathrm{ATCC} 33616^{\mathrm{T}}$ (replacement strain deposited in 1984 and approved)

$\mathrm{PC1}^{\mathrm{T}} \rightarrow$ PDDCC (= ICMP) 8561 (replacement strain deposited in 1984 but not approved; reported as incorrect type strain [4])

On the basis of this information, there is no doubt that strains ICMP 4289T, ICMP 4290, and ICMP 4291 were mislabeled by the PDDCC sometime between 1975 when the cultures were deposited and 1978 when the preprint catalog was prepared with the PDDCC strain designations. The conclusions of Hu et al. (4) that strains "ICMP 4289", ICMP 4290 , and ICMP 4291 were peritrichous and the replaced type culture ICMP 8561 was not representative"' support this possibility. Hu et al. presented no data concerning the flagellation of these strains, and made only a brief statement about this characteristic in their discussion. Furthermore, $\mathrm{Hu}$ et al. concluded that "these strains should be affiliated to Agrobacterium" with essentially no convincing explanation.

It is also evident that a labeling mistake was made by the NCPPB because the NCPPB curator and I confirmed that strain NCPPB $2982^{\mathrm{T}}$ had a polar flagellum but was different from strain $\mathrm{PC1}^{\mathrm{T}}$ in selected prominent properties (hydrolysis of starch and egg yolk). This culture is strain ICMP $4289^{\mathrm{T}}$, which was transferred from the PDDCC to the NCPPB sometime after 1975. However, the fact that strain ATCC $33616^{\mathrm{T}}$, which was transferred in 1982 from the NCPPB with the designation NCPPB $2982^{\mathrm{T}}$, was peritrichous implies that a labeling mistake may have occurred at the NCPPB sometime after strain NCPPB $2982^{\mathrm{T}}$ was transferred to the ATCC, although no direct comparison of peritrichous strains ICMP $4289^{\mathrm{T}}$ and ATCC $33616^{\mathrm{T}}$ has been conducted.

Because strain ATCC $33616^{\mathrm{T}}$, which was replaced in 1984 , was confirmed to be identical to type strain PC1 by the depositor approval check, replacement strain ICMP 8561 should also be identical to the type strain unless further mislabeling occurred in the collection. Thus, the conclusions that were drawn by $\mathrm{Hu}$ et al. (4) were not based on the facts in relation to the real taxonomic nature of this bacterium. The proposal of $\mathrm{Hu}$ et al. that strain ICMP 4289 should be designated the type strain was entirely erroneous and brought about further unnecessary confusion.

If bacterial cultures received from any culture collection are not in accordance with the original descriptions, it should be a natural step for bacterial taxonomists to question and check the identity and/or purity of the cultures. This is particularly true when the source of the cultures can be traced back to the original workers. The mislabeling of $P$. cissicola might not have become known if the ATCC curator had not checked the flagellation of strain ATCC $33616^{\mathrm{T}}$ and questioned the original author about this characteristic. The results of the ATCC's depositor approval check system subsequently demonstrated that the conclusion of $\mathrm{Hu}$ et al. (4) that strain "ICMP 8561 was incorrectly identified" was ill-conceived and was based simply on an unfounded proposition that only the cultures in the collection of $\mathrm{Hu}$ et al. were authentic.

It is difficult to understand why $\mathrm{Hu}$ et al. (4) came to their conclusions without confirming the identity of the bacterium either by conducting pathogenicity tests with the strains or by comparing the strains with the authentic cultures maintained in the Laboratory of Plant Pathology at Shizuoka University. Actually, $\mathrm{Hu}$ et al. did not even refer to the original descriptions of the bacterium. If they had carefully taken any of these steps, they would have become aware of problems with identification of their strains.

The circumstances described above have led to the possibility that the taxonomic conclusions described in the work of De Vos et al. (1) were based on the wrong strains (strains NCPPB $2982^{\mathrm{T}}$ and PDDCC 4290). Although the generic affiliation of $P$. cissicola is subject which has no direct relevance to the matter of identification under consideration here, the possibility that the wrong strains were used could seriously affect the conclusions of De Vos et al. Furthermore, a mislabeling involves two bacteria in most cases. Therefore, the present situation implies that the real cultures of $P$. cissicola have erroneously been labeled and preserved under the name of another bacterium in the ICMP.

Recent taxonomic studies of plant-pathogenic bacteria 
that have been based on numerical, chemical, and molecular analyses in most cases, have not paid attention to the identification of the bacteria. The conclusions drawn from these studies are essentially dependent on the reliability of the labels attached by the culture collections.

It should be emphasized, therefore, that all scientists who engage in taxonomic work with plant-pathogenic bacteria should check the identity and purity of the bacterial strains that they use through a preliminary examination of some prominent bacteriological traits or inoculation tests with the original host plants. This is particularly true when the cultures are provided from culture collections that do not have a depositor approval check system in the deposition process. This confirmation of identification may not be so critical when the cultures are provided by the scientists who originally described the bacteria or by the ATCC after the depositor approval check system was established.

The case of $P$. cissicola is exactly what Leifson (5) in 1966 warned the curators of type culture collections to guard against. It is regretable that his warning has to be repeated here and that the importance of the identity and purity of the cultures preserved in type culture collections has to be emphasized. The international type culture collections, such as those referred to in this paper, should be urged to use a depositor approval check system to retain their reputations for the accurate identification and culture purity. Scientists who deposit microorganisms in culture collections should be asked to cooperate with the curators for successful functioning of the system, and also the curators' careful skills in preservation of taxonomically defined, invaluable microbial resources should be appraised by the scientific community and rewarded professionally.

\section{ADDENDUM IN PROOF}

At the proofreading stage of this article, another puzzling article written by the NPCCB curator appeared in the International Journal of Systematic Bacteriology (D. E. Stead, Int. J. Syst. Bacteriol. 42:281-295, 1992). In this paper, he concluded that $P$. cissicola strains NCPPB $2982^{\mathrm{T}}$ and ICMP 8561 had identical fatty acid profiles and differed from strains ICMP 4290 and ICMP 4291, which were typical of Agrobacterium spp. These results indicate that the
NCPPB had had three different bacteria under the same code number of NCPPB $2982^{\mathrm{T}}$, namely a peritrichous strain which was sent to the ATCC in 1982, a polarly flagellated but phenotypically different strain that was confirmed by the curator in 1983, and the strain in the paper mentioned above. To avoid unnecessary confusion, curators of international culture collections should describe the history of type strains whenever they replace other strains.

\section{REFERENCES}

1. De Vos, P., M. Goor, M. Gillis, and J. De Ley. 1985. Ribosomal ribonucleic acid cistron similarities of phytopathogenic Pseudomonas species. Int. J. Syst. Bacteriol. 35:169-184.

2. Dye, D. W. 1974. The problem of nomenclature of the plant pathogenic pseudomonads. Rev. Plant Pathol. 53:953-962.

3. Goto, M., and T. Makino. 1977. Emendation of Pseudomonas cissicola, the causal organism of bacterial leaf spot of Cayratia japonica (Thunb.) Gagn. and designation of the neotype strain. Ann. Phytopath. Soc. Jpn. 43:40-45.

4. Hu, F.-P., J. M. Young, and C. M. Triggs. 1991. Numerical analysis and determinative tests for nonfluorescent plant-pathogenic Pseudomonas spp. and genomic analysis and reclassification of species related to Pseudomonas avenae Manns 1909. Int. J. Syst. Bacteriol. 41:516-525.

5. Leifson, E. 1966. Bacterial taxonomy: a critique. Bacteriol. Rev. 30:257-266.

6. New Zealand Department of Scientific and Industrial Research. 1978. Catalogue of PDDCC, preprint ed. Culture Collection of Plant Pathogenic Division, New Zealand Department of Scientific and Industrial Research, Auckland, New Zealand.

7. New Zealand Department of Scientific and Industrial Research. 1988. Catalogue, International Collection of Microorganisms from Plants, accessions 1-9519. Plant Diseases Division, New Zealand Department of Scientific and Industrial Research Auckland, New Zealand.

8. Pienta, P. A. 1983. Personal communication.

9. Skerman, V. B. D., V. McGowan, and P. H. A. Sneath (ed.). 1980. Approved lists of bacterial names. Int. J. Syst. Bacteriol. 30:225-420.

10. Stead, D. 1983. Personal communication.

11. Takimoto, S. 1939. Bacterial leaf spot of "Binboukazura." Ann. Phytopath. Soc. Jpn. 9:41-43. (In Japanese.)

12. The Williams \& Wilkins Co. 1948. Bergey's manual of determinative bacteriology, 6th ed. The Williams \& Wilkins Co., Baltimore. 